\title{
SULFETOS DO COMPLEXO GABROICO CANINDÉ, SISTEMA OROGÊNICO SERGIPANO
}

\author{
SULPHIDES OF THE CANINDÉ GABROIC COMPLEX, SERGIPANO OROGENIC SYSTEM
}

\author{
Fábio Bezerra DAMASCENO ${ }^{1}$, Bruno Luiz Leite MARTINS ${ }^{1}$, Danilo dos Santos \\ BARRETO $^{1}$, Luan Kellvin Canuto da MOTA ${ }^{2}$, Carlos Dinges Marques de SÁ ${ }^{1,2}$ \\ ${ }^{1}$ Universidade Federal de Sergipe. Grupo de Pesquisa em Recursos Minerais (RECMIN), Programa de Pós Graduação em \\ Geociências e Análise de Bacias (PGAB). Email: fbdamasceno@hotmail.com; brunogeol92@gmail.com; danilooboista@gmail.com \\ ${ }^{2}$ Universidade Federal de Sergipe /DGEOL/PGAB São Cristóvão/SE. E-mail: luankellvin@hotmail.com; dingesster@gmail.com

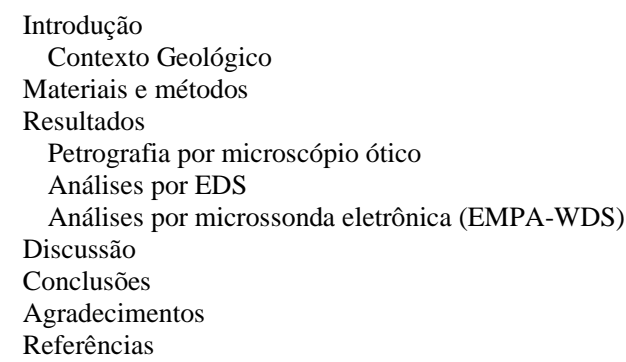

RESUMO - O Domínio Canindé, localizado na porção norte do Sistema Orogênico Sergipano, é composto por rochas metavulcânicas e metassedimentares do Complexo Canindé, sendo estas intrudidas pelo corpo gabroico denominado Complexo Gabroico Canindé. A ocorrência de mineralizações sulfetadas de Cu-Ni nos gabros já foi documentada anteriormente e será detalhada neste trabalho no que se refere à sua mineralogia e mineraloquímica, através de utilização microscopia óptica de transmissão e reflexão, de microssonda eletrônica e microscopia eletrônica de varredura. Análises por estes métodos micro analíticos permitiram identificar, em amostras coletadas nos afloramentos do Complexo Gabroico Canindé, a presença de pirita, calcopirita, pirrotita, pentlandita, esfalerita, violarita e spionkopita, com análises para os teores em elementos maiores e menores neles presentes. Entre os sulfetos identificados, a violarita $\left(\mathrm{FeNi}_{2} \mathrm{~S}_{4}\right)$ e a spionkopita $\left(\mathrm{Cu}_{39} \mathrm{~S}_{28}\right)$ ainda não haviam sido descritas anteriormente no Domínio Canindé. Evidências de alteração hidrotermal tardia nos gabros apontam para gênese dos minerais secundários violarita e spionkopita por alteração dos sulfetos primários de $\mathrm{Ni}$ e $\mathrm{Cu}$ respectivamente.

Palavras-chave: Domínio Canindé; Complexo Gabroico Canindé; Sulfetos de Cu-Ni.

\begin{abstract}
The Canindé Domain, located in the northern part of the Sergipano Orogenic System, is composed of metavulcanic and metassedimentary rocks of the Canindé Complex, which are intruded by a gabbroic body, named as the Canindé Gabbroic Complex. The occurrence of $\mathrm{Cu}-\mathrm{Ni}$ sulfide mineralization in the gabbroic rocks was already documented, and is described in detail, with respect to its mineralogy and mineral chemistry, through the utilisation of optical microscopy of transmitted and reflected light, scanning electron microscopy and electronic microprobe. The analyses by these micro-analytical methods permitted us to identify pyrite, chalcopyrite, pyrrhotite, pentlandite, sphalerite, violarite $\left(\mathrm{FeNi}_{2} \mathrm{~S}_{4}\right)$ and spionkopita $\left(\mathrm{Cu}_{39} \mathrm{~S}_{28}\right)$ in the samples of gabbros which were collected on outcrops of the Canindé Gabbroic Complex. Violarite and spionkopite were not previously described in the Canindé Domain. Evidences of late hydrothermal alteration of the gabbros point to the genesis of the secondary minerals violarite and spionkopite by alteration of the primary $\mathrm{Ni}$ and $\mathrm{Cu}$ sulfides respectively.
\end{abstract}

Keywords: Canindé Domain; Gabbros; Cu-Ni Sulfides.

\section{INTRODUÇÃO}

O Complexo Gabroico Canindé (CGC) é um corpo intrusivo às rochas do Domínio Canindé (DC), Sistema Orogênico Sergipano, localizado a sul da Província Borborema, Nordeste do Brasil. As mineralizações de cobre e níquel do CGC ocorrem de forma disseminada nos gabros, formando ocasionalmente, massas de minério irregulares (Seixas \& de Moraes, 1996). Segundo Santos et al. (2001), a mineralogia opaca é constituída por pentlandita, calcopirita, pirita, pirrotita, calcocita, covellita, ilmenita e magnetita. Os estudos publicados sobre estas ocorrências são escassos, sendo a maior parte descrições encontradas em relatórios internos da CPRM (Silva Filho et al., 1977, 1979; Tesch, 1980, Seixas \& de Moraes, 1996) ou trabalhos de geologia regional, onde estas informações são apresentadas de forma genérica (Santos \& Souza, 1988; Santos et al., 2001). Novos dados sobre as mineralizações do CGC têm sido produzidos pelo Grupo de Pesquisa em Recursos Minerais da Universidade Federal de Sergipe (RECMIN/ UFS) (e.g. Marques de Sá et al., 2018), onde este artigo servirá de subsídio a este tema expondo 
resultados das pesquisas mineralógicas dos sulfetos. Neste são apresentados e discutidos novos dados sobre os sulfetos de gabros do Complexo Gabroico Canindé.

\section{Contexto Geológico}

O Sistema Orogênico Sergipano (SOS) (Conceição et al., 2015), situa-se na parte sul da Província Borborema (NE do Brasil), é um polígono crustal metavulcanossedimentar alinhado segundo WNW-ESE, que se encontra entre o Maciço Pernambuco-Alagoas e o Cráton de São Francisco (D’el Rey Silva, 1995).

O SOS divide-se em cinco domínios geológicos (Oliveira et al., 2010): Estância, Vaza Barris, Macururé, Poço Redondo-Marancó e o Domínio Canindé. O DC é o mais setentrional deles (Figura 1), sendo constituído pelas rochas metavulcânicas e metassedimentares do Complexo Canindé (CC), que apresentam metamorfismo variando de fácies xisto-verde a fácies anfibolito, e que são intrudidas por um corpo gabroico denominado Complexo Gabroico Canindé (Silva Filho et al., 1977; Santos et al., 2001; Oliveira et al., 2010).
Silva Filho et al. (1979) individualizaram o DC em diferentes unidades. Desse modo, no Complexo Canindé, foram distinguidas as unidades: Mulungu, Garrote, Novo Gosto e Gentileza, compostas de rochas metavulcânicas, metassedimentares e corpos intrusivos. O CGC apresenta grande variedade de rochas, sendo identificados por Silva Filho et al. (1979), Tesch (1980) e Santos \& Souza (1988), gabros, noritos, microgabros, olivinagabros, leucogabros, anortositos, troctolitos e outras rochas ultramáficas. Tesch (1980) observou ainda que as principais ocorrências de sulfetos estão associadas aos troctolitos.

O CGC é um dos dois corpos intrusivos máfico-ultramáficos com mineralizações de $\mathrm{Cu}$ $\mathrm{Ni}$ conhecidos no SOS. O mais estudado é o Complexo Serrote da Laje localizado no estado de Alagoas, que se encontra em atividade de extração de $\mathrm{Cu}$; O outro é o CGC, que até o presente nível de conhecimento e interesse de mercado é considerado sub-econômico, apresentando teores de cobre entre 0,13 e $0,34 \%$ e de níquel entre 0,17 e $0,35 \%$, segundo o estudo realizado por Tesch (1980).

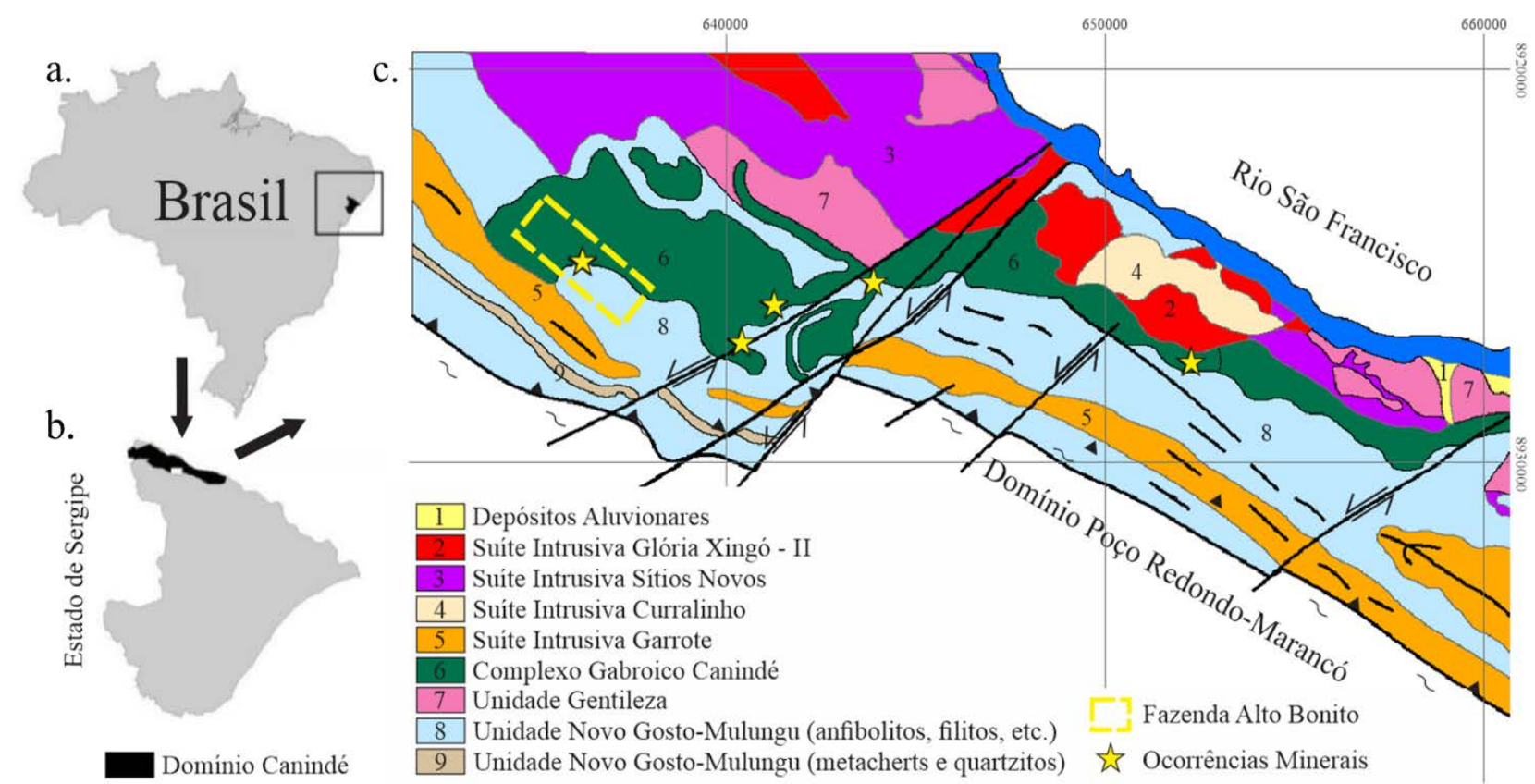

Figura 1 - a. Mapa do Brasil e localização do Estado de Sergipe; b. Área do Domínio Canindé (DC); c. Mapa Geológico da porção sul-central do DC com localização da área de estudo na Fazenda Alto Bonito (adaptado de Teixeira, 2014).

\section{MATERIAIS E MÉTODOS}

Foi efetuado estudo cartográfico utilizando o software Google Earth Pro juntamente com a cartografia geológica realizada pela CPRM (Tesch, 1980; Santos \& Souza, 1988; Santos, 2001, Teixeira, 2014). Em campanhas de campo foram coletadas cinquenta amostras de gabros com mineralização disseminada de sulfetos no afloramento da Fazenda do Alto Bonito (Figura 1).

Sete lâminas delgadas polidas foram preparadas com o auxílio dos laboratórios da Superintendência de Geologia de Salvador, do Serviço Geológico do Brasil (CPRM-Bahia). Três superfícies polidas foram preparadas no Condomínio de Laboratórios Multiusuários das Geociências - Universidade 
Federal de Sergipe (CLGeo/UFS). As preparações foram analisadas no CLGeo em microscópio óptico (MO) de luz transmitida e refletida da marca OPTON modelo TNP-09NT. Para realizar as microfotografias utilizou-se um microscópio petrográfico Olympus modelo BX41 com câmera digital Olympus SC30.

Para identificação de cristais sub-microscópicos e análise dos teores de elementos maiores e menores utilizou-se o Microscópio Eletrônico de Varredura (MEV) do CLGeo, de marca Tescan modelo Vega 3, com detectores de elétrons secundários (ES), elétrons retroespalhados (ER) e espectrômetro de energia dispersiva (EDS) de marca Oxford Instruments, modelo X-Act. As condições no momento da análise foram: vácuo de $1,5 \times 10^{-3} \mathrm{~Pa}$; tensão do feixe de $15 \mathrm{kV}$; corrente 20 nA e diâmetro do feixe de $400 \mathrm{~nm}$. O tempo médio de contagem foi de 30 segundos.

As análises por microssonda eletrônica (EMPA-WDS) foram realizadas no Laboratório Nacional de Energia e Geologia (LNEG), em Porto, Portugal. Foi utilizada uma microssonda eletrônica JEOL JXA 8500-F. As análises foram realizadas nas seguintes condições: tensão de 20 $\mathrm{kV}$ e intensidade de corrente do feixe de $20 \mathrm{nA}$, sendo utilizados padrões reais para cada elemento e feitas as correções ZAF.

\section{RESULTADOS}

As características petrográficas das amostras analisadas são muito semelhantes. Desse modo as sete lâminas analisadas foram descritas conjuntamente, para os diferentes métodos de análise utilizados, MO, EDS-MEV e EMPA-WDS.

\section{Petrografia por Microscópio Óptico}

As amostras em estudo são gabros com textura isotrópica, inequigranulares, definida por fenocristais de plagioclásio, apresentando minerais fortemente alterados e fraturados. Os minerais, quanto à forma, ocorrem de anédricos a subédricos. A mineralogia principal é composta por plagioclásio, piroxênio e anfibólio, apresentando como minerais de alteração sericita, calcita, clorita e minerais opacos.

Os sulfetos, opacos à luz transmitida, ocorrem como minerais acessórios, sendo identificados por microscopia de luz refletida os minerais: pirita, calcopirita e pirrotita. Os demais sulfetos foram identificados com EDS.

A labradorita encontra-se fraturada e às vezes alterada, apresentando-se como cristais subédricos e anédricos, compondo a matriz da rocha ou ocasionalmente como inclusões em piroxênio. Estão geminados segundos as leis de albita e albita-Carlsbad.

Os cristais variam de $0,5 \mathrm{~mm}$ a $9,22 \mathrm{~mm}$ e apresentam contatos irregulares com os outros minerais, sendo exceção os ocasionais contatos retos com o piroxênio. Encontram-se frequentemente alterados para sericita e calcita, esta que ocorre também em fraturas (Figura 2a).

O clinopiroxênio ocorre como cristais anédricos e subédricos. Ocasionalmente ocorrem cristais de plagioclásio e de minerais opacos inclusos (Figura 2b). Por vezes, apresentam extinção ondulante e estão fraturados e frequen- temente alterados para anfibólio (Figura 2c).

Os tamanhos variam de $0,3 \mathrm{~mm}$ à $8,2 \mathrm{~mm}$ e fazem contatos retos a irregulares com plagioclásio e irregulares com os restantes minerais da rocha.

O anfibólio se apresenta como cristais subédricos e anédricos, em geral como produto da alteração do clinopiroxênio (Figura 2d). Predomina a hornblenda, mas tremolita/actinolita também são observadas em quantidades ínfimas. Os cristais de hornblenda têm tamanhos que variam de $0,2 \mathrm{~mm}$ à $2,8 \mathrm{~mm}$, apresentam contatos irregulares com os outros minerais e alteram para clorita.

Os minerais opacos, posteriormente estudados por microscopia de reflexão, ocorrem variando de euédricos e anédricos, dispersos, inclusos ou intersticiais. Os cristais variam de $0,04 \mathrm{~mm}$ a $1,9 \mathrm{~mm}$.

De modo geral apresentam contatos irregulares com os demais minerais da lâmina. Aparecem frequentemente como inclusões no plagioclásio ou por vezes no piroxênio e estão frequentemente oxidados (Figura 2e).

Por microscopia de luz refletida foi possível identificar calcopirita e pirita. Verificou-se que a pirita se apresenta de anédrica e euédrica, de cor branca amarelada e geralmente apresentando oxidação nas bordas dos cristais, e que a calcopirita é sempre anédrica, por vezes intersticial, de cor amarela e ocorre tanto em contato com a pirita como isolada na matriz (Figura 2f).

\section{Análises por EDS}

As análises por espectrometria de energia dispersiva acoplado ao microscópio eletrônico de varredura permitiram identificar os seguintes minerais: pirita, calcopirita, pirrotita, pentlandita, esfalerita, violarita e spionkopita. 

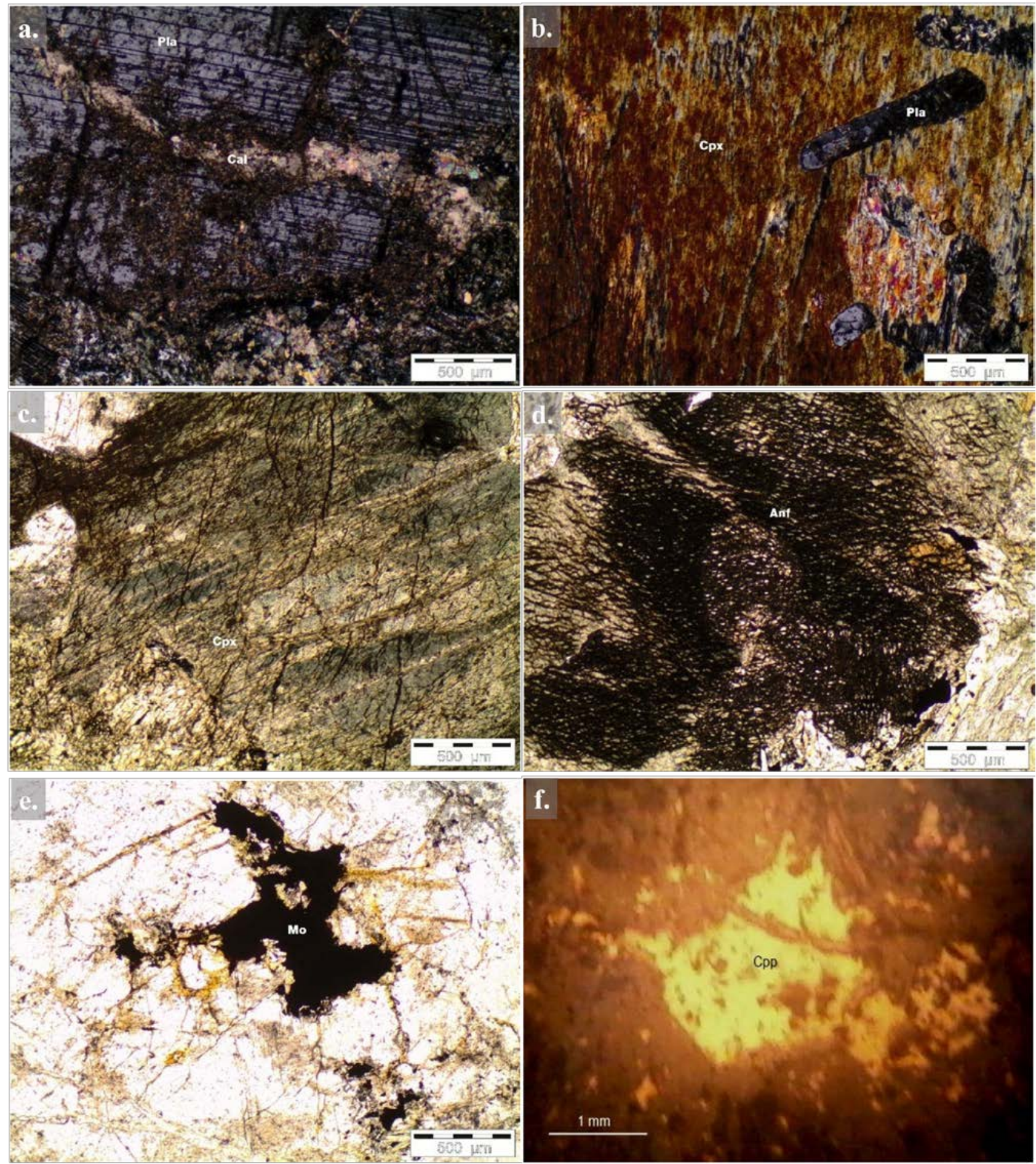

Figura 2 - a) Cristal de plagioclásio alterado, preenchido por calcita nas fraturas. b) Cristal de clinopiroxênio com inclusão de plagioclásio. Nicóis cruzados. c) Cristal de clinopiroxênio alterando para clorita. Nicóis cruzados. d) Hornblenda de alteração do clinopiroxênio. e) Mineral opaco intersticial com oxidação nas bordas. Nicóis paralelos. f) Cristal de calcopirita imerso em hornblenda. Luz refletida. $\mathrm{Cal}=$ Calcita, $\mathrm{Pl}=$ Plagioclásio, $\mathrm{Cpx}=$ Clinopiroxênio, Amp $=$ Anfibólio. Mo $=$ Mineral Opaco, Cpp $=$ Calcopirita.

As imagens de elétrons retroespalhados obtidas a partir de um detector acoplado ao MEV, retrataram os aspectos texturais sub-microscópicos, sendo os teores de elementos maiores analisados por EDS.

A pirita (Figura 3a) é o sulfeto mais abundante. Os seus cristais ocorrem euédricos e anédricos. Das análises apresentadas na tabela 1 verifica-se que a média da sua composição é de Fe 41,0 wt.\% e S 57,5 wt.\%, apresentando teores de outros elementos menores como Ni, Co e As.

Nas análises aos cristais de pirita foi possível notar os seguintes aspectos: elevado teor de enxofre; elevados teores de elementos menores, como por exemplo os detectados para o cobalto, que ultrapassam 2 wt.\% (espectros S1 e S25), o 
arsênio, com teores de 0,8 e 0,9 wt.\% (espectros S1 e S3) e o níquel, encontrado na análise do espectro S40, com teor de 2,7 wt.\%. O cálculo da formula estrutural da pirita, tem como resultado: $\mathrm{Ni}_{0,01} \mathrm{Co}_{0,02} \mathrm{Fe}_{0,82} \mathrm{~S}_{2}$.

Tabela 1 - Análises de pirita nas amostras FAB1e FAB7, $\mathrm{n}=6$, teores em wt. $\%$.

\begin{tabular}{l|c|c|c|c|c|c}
\hline Amostra & S & Fe & Co & As & Ni & Total \\
\hline FAB1-S1 & 56,9 & 40,2 & 2,1 & 0,8 & 0,0 & $\mathbf{1 0 0}$ \\
\hline FAB1-S2 & 57,5 & 42,1 & 0,4 & 0,0 & 0,0 & $\mathbf{1 0 0}$ \\
\hline FAB1-S3 & 58,4 & 40,7 & 0,0 & 0,9 & 0,0 & $\mathbf{1 0 0}$ \\
\hline FAB1-S40 & 56,2 & 41,2 & 0,0 & 0,0 & 2,7 & $\mathbf{1 0 0}$ \\
\hline FAB7-S12 & 60,0 & 40,0 & 0,0 & 0,0 & 0,0 & $\mathbf{1 0 0}$ \\
\hline FAB7-S25 & 55,7 & 42,1 & 2,2 & 0,0 & 0,0 & $\mathbf{1 0 0}$ \\
\hline Média & 57,5 & 41,0 & 0,8 & 0,3 & 0,4 & \\
\hline
\end{tabular}

A calcopirita, também abundante, ocorre predominantemente sob forma anédrica, preenchendo interstícios e eventualmente em cristais subédricos (Figura 3d). Este mineral foi identificado em 27 análises por EDS.

Os resultados das análises em cristais de calcopirita são apresentados na tabela 2, sendo as médias dos teores por percentual em peso dos elementos maiores: $\mathrm{Cu} 37,5$ wt.\%, Fe 28,0 wt.\% e S 34,6 wt.\%. Observa-se variação considerável nos teores de elementos maiores (Tabela II). O cálculo da fórmula estrutural, para o valor médio das concentrações dos elementos resultou na fórmula: $\mathrm{Fe}_{0,93} \mathrm{Cu}_{1,09} \mathrm{~S}_{2}$.

A pirrotita ocorre anédrica, como ilustrada na Figura 4c, identificada a partir dos resultados de 8 análises, apresentadas na tabela 3. As médias dos teores dos elementos são: Fe 54,8 wt.\%, Ni 0,5 wt.\%, Co 0,3 wt.\% e S 44,3 wt.\%. Para o cálculo da fórmula estrutural, foram considerados os valores médios das concentrações dos elementos, resultando na fórmula $\mathrm{Ni}_{0,01} \mathrm{Fe}_{0,71} \mathrm{~S}_{1}$.

A pentlandita encontra-se alterada nas bordas dos cristais, passando a violarita, conforme ilustrado na figura 3c. A pentlandita foi apenas identificada nas amostras FAB 1 e 7.

A violarita é um mineral frequentemente nestas amostras e resulta da alteração da pentlandita através de um processo de oxidação. Foram realizadas 4 análises por EDS-MEV em violarita (espectros 2, 3 e 4 da figura 13 e espectro S41), cujos resultados se encontram na tabela 4. As médias dos teores dos elementos maiores foram Ni 40,2 wt.\%; Fe 15,1 \% e S 44,8 wt.\%. O cálculo da fórmula estrutural foi realizado com os teores médios de Fe, Ni e S das quatro análises onde a violarita foi identificada, resultando na fórmula $\mathrm{Fe}_{0,77} \mathrm{Ni}_{1,96} \mathrm{~S}_{4}$.

Tabela 2 - Análises de calcopirita nas amostras FAB1 e FAB7, $n=27$, teores em wt.\%.

\begin{tabular}{|c|c|c|c|c|}
\hline Amostra & S & $\mathrm{Cu}$ & Fe & Total \\
\hline FAB1-S1 & 27,9 & 34,5 & 37,6 & 100 \\
\hline FAB1-S6 & 35,5 & 36,8 & 27,7 & 100 \\
\hline FAB1-S8 & 23,6 & 43,6 & 32,8 & 100 \\
\hline FAB1-S8 & 35,1 & 39,1 & 25,7 & 100 \\
\hline FAB1-S18 & 34,7 & 35,1 & 30,1 & 100 \\
\hline FAB1-S21 & 35,4 & 35,6 & 28,9 & 100 \\
\hline FAB1-S24 & 34,8 & 38,9 & 26,4 & 100 \\
\hline FAB1-38 & 35,0 & 27,9 & 37,1 & 100 \\
\hline FAB1-39 & 34,9 & 28,1 & 37,0 & 100 \\
\hline FAB1-S63 & 34,2 & 38,2 & 27,6 & 100 \\
\hline FAB1-S64 & 34,6 & 38,4 & 27,1 & 100 \\
\hline FAB1-S65 & 35,1 & 38,0 & 0 & 100 \\
\hline FAB1-S66 & 36,3 & 37,4 & 26,3 & 100 \\
\hline FAB1-S67 & 35,2 & 38,4 & 26,4 & 100 \\
\hline FAB1-S68 & 35,2 & 38,2 & 26,6 & 100 \\
\hline FAB1-S76 & 34,8 & 37,7 & 27,5 & 100 \\
\hline FAB1-S77 & 35,3 & 38,2 & 26,4 & 100 \\
\hline FAB1-S78 & 35,5 & 38,8 & 25,7 & 100 \\
\hline FAB1-S80 & 34,8 & 37,9 & 27,3 & 100 \\
\hline FAB1-S81 & 36,0 & 37,0 & 27,0 & 100 \\
\hline FAB1-S82 & 35,9 & 37,1 & 27,0 & 100 \\
\hline FAB7-S2 & 35,9 & 44,5 & 19,6 & 100 \\
\hline FAB7-S23 & 35,2 & 39,3 & 25,5 & 100 \\
\hline FAB7-S59 & 35,6 & 38,5 & 25,9 & 100 \\
\hline FAB7-S60 & 35,5 & 38,5 & 26,0 & 100 \\
\hline FAB7-S75 & 36,2 & 37,4 & 26,4 & 100 \\
\hline FAB7-S77 & 35,4 & 38,3 & 26,3 & 100 \\
\hline Média & 34,6 & 37,5 & 28,0 & \\
\hline
\end{tabular}

Tabela 3 - Análises de pirrotita na amostra FAB, $\mathrm{n}=5$, teores em wt.\%.

\begin{tabular}{c|c|c|c|c|c}
\hline Amostra & S & Fe & Co & Ni & Total \\
\hline FAB1-S17 & 44,4 & 55,6 & 0,0 & 0,0 & $\mathbf{1 0 0 , 0}$ \\
\hline FAB1-S23 & 44,7 & 55,3 & 0,0 & 0,0 & $\mathbf{1 0 0 , 0}$ \\
\hline FAB1-S25 & 44,5 & 55,5 & 0,0 & 0,0 & $\mathbf{1 0 0 , 0}$ \\
\hline FAB1-36 & 42,2 & 57,8 & 0,0 & 0,0 & $\mathbf{1 0 0 , 0}$ \\
\hline FAB1-S69 & 43,4 & 51,8 & 3,0 & 1,8 & $\mathbf{1 0 0 , 0}$ \\
\hline FAB1-S70 & 43,1 & 55,2 & 0,0 & 1,8 & $\mathbf{1 0 0 , 0}$ \\
\hline FAB7-S3 & 46,3 & 53,5 & 0,0 & 0,0 & $\mathbf{9 9 , 8}$ \\
\hline FAB7-S5 & 45,3 & 53,2 & 0,0 & 1,3 & $\mathbf{9 9 , 8}$ \\
\hline FAB7-S43 & 45,0 & 55,0 & 0,0 & 0,0 & $\mathbf{1 0 0 , 0}$ \\
\hline Média & 44,32 & 54,76 & 0,33 & 0,53 &
\end{tabular}

1A calcopirita pode apresentar alteração nas bordas dos cristais para spionkopita $\left(\mathrm{Cu}_{39} \mathrm{~S}_{28}\right)$ 
(Figura 3b). Este sulfeto de cobre foi identificado na amostra FAB17, em 4 pontos analisados nas bordas de um cristal de calcopirita (Tabela 5).

Tabela 4 - Análises de violarita nas amostras FAB, $\mathrm{n}=4$, teores em wt.\%.

\begin{tabular}{l|c|c|c|c}
\hline \multicolumn{1}{|c}{ Amostra } & S & Ni & Fe & Total \\
\hline FAB1-S41 & 46,0 & 37,3 & 16,8 & $\mathbf{1 0 0}$ \\
\hline FAB7-S2 & 43,8 & 42,5 & 13,7 & $\mathbf{1 0 0}$ \\
\hline FAB7-S3 & 46,4 & 39,1 & 14,5 & $\mathbf{1 0 0}$ \\
\hline FAB7-S4 & 42,9 & 41,8 & 15,3 & $\mathbf{1 0 0}$ \\
\hline Média & 44,8 & 40,2 & 15,1 & \\
\hline
\end{tabular}

As médias dos teores dos elementos maiores encontrados na spionkopita são: $\mathrm{Cu}$ 66,0 wt.\%, Fe 5,0 wt.\% e S 28,9 wt.\%. Para o cálculo da fórmula estrutural foram utilizados os valores da concentração dos elementos $\mathrm{Fe}, \mathrm{Cu}$ e $\mathrm{S}$ do espectro S71, atribuindo a $\mathrm{S}$ o valor 28, para este espectro têm-se a fórmula $\mathrm{Cu}_{37,05} \mathrm{Fe}_{4,32} \mathrm{~S}_{28}$.

Identificou-se também a ocorrência de dois cristais de esfalerita na amostra FAB31, onde estão em contato com cristais de calcopirita. As

análises mostram que além dos teores em Zn e S, outros elementos também estão presentes, como o Fe que varia entre 3,4 e 5,6 wt.\% e o Cd entre 1,59 e 1,64 wt.\% (Tabela 6). O cálculo da fórmula estrutural foi relativo ao espectro S31, resultando na fórmula: $\mathrm{Fe}_{0,06} \mathrm{Cd}_{0,01} \mathrm{Zn}_{0,85} \mathrm{~S}_{1}$.

Tabela 5 - Análises de spionkopita nas amostras FAB 17, $\mathrm{n}=4$, teores em wt.\%.

\begin{tabular}{l|c|c|c|c}
\hline \multicolumn{1}{c|}{ Amostra } & Cu & S & Fe & Total \\
\hline FAB17-70 & 65,6 & 32,3 & 2,1 & $\mathbf{1 0 0}$ \\
\hline FAB17S71 & 67,4 & 25,7 & 6,9 & $\mathbf{1 0 0}$ \\
\hline FAB17S72 & 64,4 & 28,2 & 7,1 & $\mathbf{9 9 , 7}$ \\
\hline FAB17S73 & 66,7 & 29,4 & 3,9 & $\mathbf{1 0 0}$ \\
\hline Média & 66,0 & 28,9 & 5,0 & \\
\hline
\end{tabular}

Tabela 6 - Análises de esfalerita nas amostras FAB31, $\mathrm{n}=2$, teores em wt.\%.

\begin{tabular}{c|c|c|c|c|c}
\hline Amostra & Zn & S & Fe & Cd & Total \\
\hline S30 & 57,5 & 35,3 & 5,6 & 1,6 & $\mathbf{1 0 0}$ \\
\hline S31 & 60,3 & 34,6 & 3,4 & 1,6 & $\mathbf{1 0 0}$ \\
\hline Média & 58,9 & 35,0 & 4,5 & 1,6 & \\
\hline
\end{tabular}
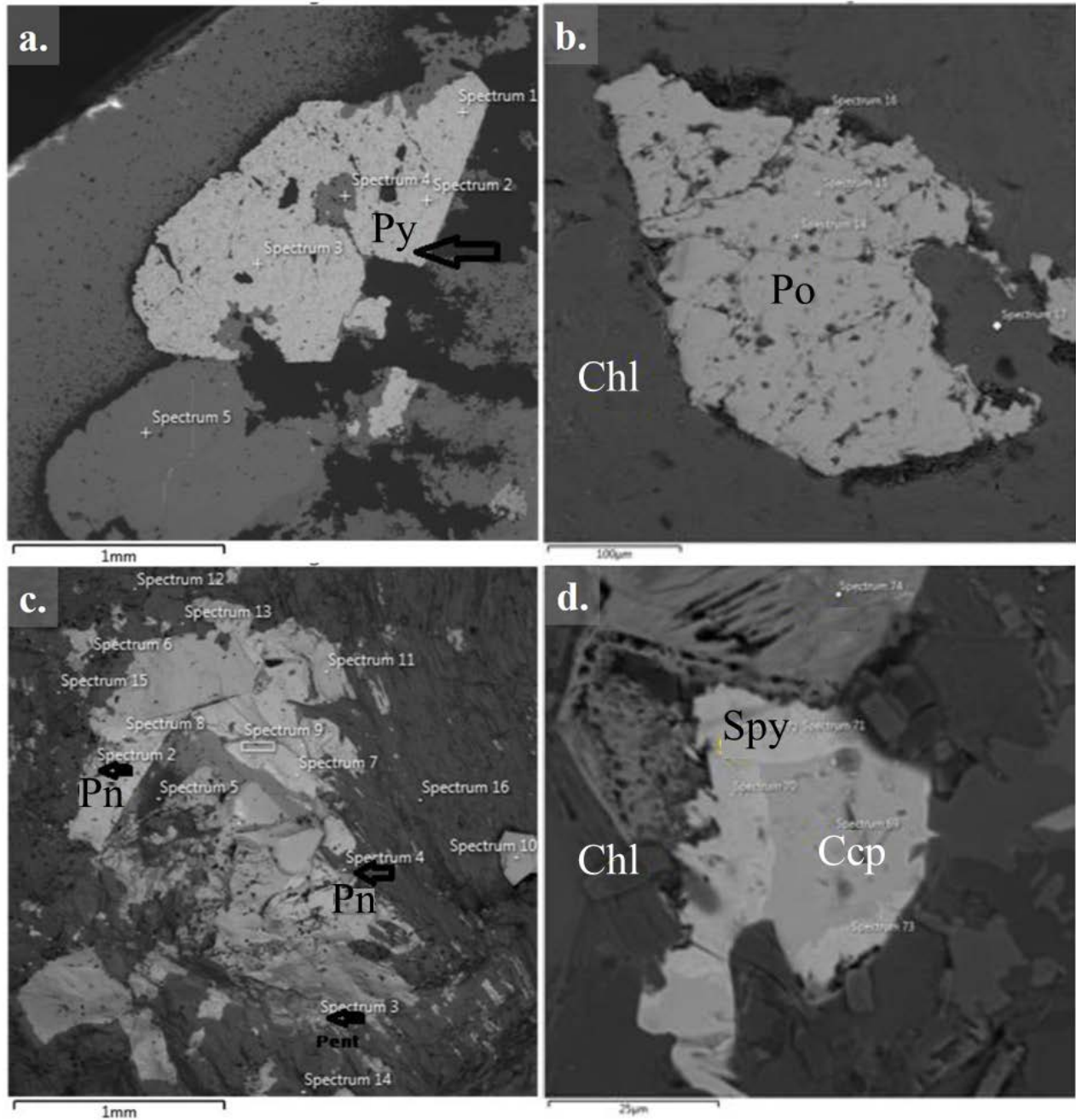

Figura 3 - Imagens de ER. a.) Cristal de pirita. b.) Cristal de pirrotita. c.) Cristais de pentlandita alterados para violarita nas bordas. d.) Cristais de calcopirita com bordas alteradas para spionkopita. Py = Pirita; Po = Pirrotita; Chl = Clorita; Spy = Spionkopita; Pn = Pentlandita . 
Análises Por Microssonda Eletrônica (EMPAWDS)

A partir das análises de microssonda eletrônica foi possível identificar os elementos e quantificar os teores das amostras FAB analisadas por esta técnica. Estas análises permitiram quantificar uma diversidade de elementos traço que ocorrem em concentrações inferiores a 0,1 wt.\%. Foram analisados os seguintes minerais: pirita, calcopirita e pirrotita.

A pirita é o sulfeto mais abundante, tendo-se realizado um total de 9 análises. Das análises, conforme apresentadas na tabela 7 , verifica-se que a média da concentração dos principais elementos de sua composição é de $\mathrm{Fe} 44,74$ wt.\%, e S 53,45 wt.\%. Para o cálculo da fórmula estrutural, utilizou-se o valor de concentração média de cada um dos elementos, resultando na fórmula

$\mathrm{Ni}_{0,001} \mathrm{As}_{0,006} \mathrm{Bi}_{0,001} \mathrm{Co}_{0,015} \mathrm{~Pb}_{0,001} \mathrm{Fe}_{0,961} \mathrm{~S}_{2}$. Os elementos Se, In, Mn, Ag, Cr, Cd, Zn, V e Pt foram identificados, porém os seus teores são, de uma forma geral, abaixo do limite de detecção de
0,01\%.

A calcopirita (Tabela 8), tem a sua composição média de Fe 28,99 wt.\%, Cu 34,35 wt.\% e S 34,79 wt.\%.

A fórmula estrutural a partir do valor da média das concentrações dos elementos é $\mathrm{As}_{0,001} \mathrm{Bi}_{0,001} \mathrm{Cr}_{0,002} \mathrm{Co}_{0,002} \mathrm{Zn}_{0,001} \mathrm{~Pb}_{0,001} \mathrm{Fe}_{0,957} \mathrm{Cu}_{0}$ , $996 \mathrm{~S}_{2}$. Os demais elementos identificados, como Se, In, Ni, Au, Mn, Ag, Cd e V, tiveram para a fórmula estrutural valores inferiores a 0,001.

Foram realizadas 7 análises em pirrotita (Tabela 9). Verifica-se que a média da composição para os principais elementos é $\mathrm{S}$ 38,94 wt.\% Fe 57,40 wt.\%, com destaque para o Ni que apresentou um valor médio de 1,40 wt.\%. Para o cálculo da fórmula estrutural, foram considerados os valores médios das concentrações dos elementos obtendo-se a fórmula $\mathrm{Ni}_{0,020} \mathrm{Mn}_{0,004} \mathrm{Bi}_{0,001} \mathrm{Co}_{0,001} \mathrm{Fe}_{0,846} \mathrm{~S}_{1}$. Os demais elementos identificados ocorrem em concentrações muito baixas, com valores encontrados inferiores a 0,01 , entre eles o Se, In, As, Au, Ag, Cr, Cd, Zn, Pb, V e Pt.

Tabela 7 - Análises de pirita nas amostras FAB 1 e FAB 7, n = 9, teores em \%. Valores ilustrados como “-“, significa que não foram identificados.

\begin{tabular}{|c|c|c|c|c|c|c|c|c|c|c|c|c|c|c|c|c|c|c|}
\hline Amostra & Se & In & $\mathrm{Ni}$ & $\mathbf{S}$ & As & $\mathbf{F e}$ & Mn & Ag & $\mathbf{B i}$ & $\mathrm{Cr}$ & $\mathbf{C u}$ & Cd & Co & $\mathrm{Zn}$ & $\mathbf{P b}$ & $\mathbf{V}$ & Pt & Total \\
\hline FAB 1-7 & 0,01 & 0,01 & 0,09 & 53,57 & 0,07 & 45,51 & - & 0,01 & 0,14 & 0,01 & 0,03 & 0,02 & 0,10 & - & 0,11 & - & - & 99,66 \\
\hline FAB 1-8 & - & - & 0,03 & 53,21 & 0,69 & 43,69 & - & - & 0,15 & - & - & - & 1,48 & - & 0,13 & - & 0,11 & 99,48 \\
\hline FAB 1-11 & 0,01 & 0,01 & 0,06 & 53,28 & 0,78 & 44,23 & - & 0,05 & 0,22 & - & - & 0,03 & 1,30 & 0,04 & 0,14 & 0,03 & 0,14 & 100,34 \\
\hline FAB 1-12 & - & - & 0,18 & 53,86 & 0,18 & 45,00 & 0,01 & - & 0,12 & 0,03 & - & - & 0,15 & 0,03 & 0,10 & - & - & 99,67 \\
\hline FAB 1-13 & 0,02 & 0,01 & 0,03 & 53,04 & 0,49 & 44,40 & - & - & 0,17 & - & - & - & 1,12 & - & 0,12 & - & 0,04 & 99,43 \\
\hline FAB 1-15 & - & - & 0,04 & 53,68 & 0,37 & 44,43 & - & - & 0,25 & - & - & 0,03 & 0,80 & - & 0,16 & - & 0,05 & 99,80 \\
\hline FAB 1-16 & - & - & 0,03 & 54,07 & 0,26 & 43,60 & - & 0,03 & 0,12 & 0,01 & 0,04 & 0,01 & 0,78 & - & 0,12 & - & - & 99,06 \\
\hline FAB 7-2 & - & - & 0,02 & 53,44 & - & 45,94 & - & 0,01 & 0,16 & 0,01 & - & 0,02 & 0,31 & - & 0,10 & - & - & 100,01 \\
\hline FAB 7-5 & - & - & 0,01 & 52,89 & 0,36 & 45,83 & 0,01 & - & 0,13 & - & 0,01 & - & 0,83 & - & 0,12 & - & - & 100,19 \\
\hline Média & 0,00 & 0,00 & 0,05 & 53,45 & 0,36 & 44,74 & 0,00 & 0,01 & 0,16 & 0,01 & 0,01 & 0,01 & 0,76 & 0,01 & 0,12 & 0,00 & 0,04 & \\
\hline
\end{tabular}

Tabela 8 - Análises de calcopirita nas amostras FAB 1 e FAB 7, n = 5, teores em \%. Valores ilustrados como “-“, significa que não foram identificados.

\begin{tabular}{|c|c|c|c|c|c|c|c|c|c|c|c|c|c|c|c|c|c|c|}
\hline \multicolumn{19}{|c|}{ 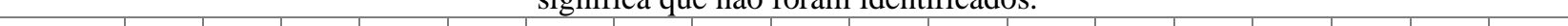 } \\
\hline Amostra & Se & In & $\mathrm{Ni}$ & $\mathbf{S}$ & As & $\mathbf{F e}$ & Au & Mn & Ag & Bi & $\mathrm{Cr}$ & $\mathbf{C u}$ & Cd & Co & $\mathrm{Zn}$ & $\mathbf{P b}$ & $\mathbf{V}$ & Total \\
\hline FAB 1-3 & 0,01 & - & - & 34,44 & 0,05 & 28,58 & 0,03 & - & - & 0,1 & 0,02 & 33,87 & - & 0,05 & 0,03 & 0,10 & - & 97,28 \\
\hline FAB 1-4 & - & 0,02 & 0,02 & 35,08 & 0,1 & 28,08 & - & - & 0,03 & 0,13 & 0,01 & 33,79 & 0,03 & 0,01 & 0,02 & 0,06 & - & 97,36 \\
\hline FAB 1-14 & 0,02 & 0,01 & - & 35,36 & 0,02 & 28,93 & - & - & - & 0,17 & 0 & 34,65 & 0,02 & 0,07 & 0,13 & 0,09 & 0,01 & 99,51 \\
\hline FAB 7-3 & - & - & - & 34,38 & 0,06 & 29,78 & - & 0,02 & 0,03 & 0,2 & 0,03 & 34,88 & - & - & - & 0,09 & 0,01 & 99,49 \\
\hline FAB 7-4 & - & - & - & 34,69 & - & 29,58 & - & 0,03 & 0,02 & 0,16 & 0,2 & 34,57 & 0,02 & 0,18 & 0,04 & 0,10 & 0,02 & 99,59 \\
\hline Média & 0,01 & 0,01 & 0,00 & 34,79 & 0,05 & 28,99 & 0,01 & 0,01 & 0,02 & 0,15 & 0,05 & 34,35 & 0,01 & 0,06 & 0,04 & 0,09 & 0,01 & \\
\hline
\end{tabular}

\section{DISCUSSÃO}

Nos resultados obtidos se observou a alteração clorita e na forte oxidação nas bordas dos produzida nos cristais de plagioclásio para calcita e sericita, de clinopiroxênio para anfibólio e sulfetos.

Estas observações evidenciam que estas 
rochas sofreram um processo de alteração hidrotermal tardio e do tipo fílico.

A partir das análises por EMPA-WDS foi possível medir de forma mais precisa os teores de elementos menores e traços para três sulfetos, a pirita, a calcopirita e a pirrotita. Os resultados tiveram grande importância ao serem comparados aos obtidos em EDS-MEV, tendo sido observada uma pequena variação nos teores de enxofre. No estudo por este método também foi identificada a presença de metais nobres e do grupo dos platinóides.

Tabela 9 - Análises de pirrotita nas amostras FAB 1 e FAB 7, n= 7, teores em \%. Valores ilustrados como “- “, significa que não foram identificados.

\begin{tabular}{|c|c|c|c|c|c|c|c|c|c|c|c|c|c|c|c|c|c|c|}
\hline Amostra & Se & In & $\mathrm{Ni}$ & S & As & $\mathbf{F e}$ & Mn & Ag & $\mathbf{B i}$ & $\mathrm{Cr}$ & $\mathbf{C u}$ & Cd & Co & Zn & $\mathbf{P b}$ & $\mathbf{V}$ & Pt & Total \\
\hline FAB 1-1 & - & 0,00 & 1,59 & 38,35 & - & 56,55 & - & 0,03 & 0,19 & 0,02 & - & 0,03 & 0,07 & 0,02 & 0,10 & 0,01 & - & 96,96 \\
\hline FAB 1-2 & 0,04 & - & 1,49 & 38,81 & 0,12 & 57,87 & - & - & 0,12 & - & - & 0,03 & 0,06 & - & 0,11 & 0,02 & - & 98,66 \\
\hline FAB 1-5 & 0,02 & - & 1,48 & 39,26 & 0,05 & 58,02 & - & 0,03 & 0,20 & - & - & 0,01 & 0,08 & - & 0,08 & - & 0,06 & 99,29 \\
\hline FAB 1-6 & - & - & 1,46 & 39,01 & - & 56,67 & - & 0,02 & 0,13 & - & 0,17 & 0,02 & 0,08 & - & 0,02 & 0,02 & - & 97,60 \\
\hline FAB 1-10 & 0,01 & 0,02 & 0,31 & 38,63 & 0,01 & 58,08 & - & 0,01 & 0,15 & - & - & - & 0,08 & - & 0,16 & 0,04 & 0,11 & 97,62 \\
\hline FAB 7-1 & - & - & 1,69 & 38,55 & - & 57,38 & 1,80 & - & 0,07 & 0,17 & 0,00 & - & 0,07 & - & 0,11 & 0,03 & - & 99,86 \\
\hline FAB 7-6 & - & - & 1,79 & 39,98 & 0,06 & 57,21 & - & - & 0,13 & 0,03 & - & 0,02 & 0,08 & - & 0,12 & 0,00 & 0,15 & 99,57 \\
\hline Média & 0,01 & 0,00 & 1,40 & 38,94 & 0,03 & 57,40 & 0,26 & 0,01 & 0,14 & 0,03 & 0,02 & 0,02 & 0,07 & 0,00 & 0,10 & 0,02 & 0,05 & \\
\hline
\end{tabular}

Segundo vários autores (e.g. Hawley \& Nichol, 1961; Dare et al., 2011), e baseados em dados de cristais de pirita analisados em diversos depósitos, a pirita magmática pode apresentar traços de Pt e enriquecimento em Co em relação à pirrotita, estando este fato de acordo com os resultados obtidos por esse estudo. Resultados que sustentam a gênese magmática também foram encontrados para os cristais de pirrotita, sendo estes de uma forma geral mais enriquecidos em $\mathrm{Ni}$ e $\mathrm{Cr}$.

A calcopirita apresenta teores de traço similares ao que se observam noutras mineralizações magmáticas (Hawley \& Nichol, 1961; Cabri \& Laflamme, 1976; Barnes \& Ripley, 2016).

A violarita e a spionkopita, pela primeira vez identificadas durante este estudo nos gabros do Domínio Canindé, são resultantes de um processo de alteração hidrotermal supergênica superimposta a pentlandita e a calcopirita respectivamente e que corresponde a uma forte oxidação.

No primeiro caso, a alteração da pentlandita em violarita, é uma transformação que ocorre em ambiente oxidante que origina uma reação entre o mineral primário, o oxigênio e ácido sulfídrico, como descrito por Tenailleau, et al. (2006).
Pentlandita $+2.75 \mathrm{O}_{2(\mathrm{~g})}+5,5 \mathrm{H}_{2} \mathrm{~S}_{(\mathrm{aq})}=2,25$ Violarita $+2,25$ Pirita $+5,5 \mathrm{H}_{2} \mathrm{O}$.

No segundo caso, observa-se que a calcopirita por vezes apresenta alteração nas bordas, onde a reação dá origem a spionkopita, resultante também de um processo de oxidação. Resultados do EDS-MEV e EMPA-WDS indicando a formação deste sulfeto enriquecido em $\mathrm{Cu}$, da Família da Covelita, foram obtidos em análises da amostra FAB17. Estas observações apontam para um processo como o descrito por Goble (1985), e sugere que a spionkopita ocorre substituindo outros sulfetos de cobre, sendo formada por uma substituição seletiva do $\mathrm{Cu}$ na rede cristalina. As variações nos teores de $\mathrm{S}$ medidas em alguns dos sulfetos devem estar relacionadas também à ação dos fluidos hidrotermais e processos de sulfurização tardios.

A sequência de cristalização, determinada a partir da associação mineral observada nas amostras estudadas, corresponde às seguintes paragêneses:

Paragênese magmática: plagioclásio - piroxênio - pirrotita - pentlandita

Paragênese tardi-magmática: hornblenda - pirita - calcopirita - esfalerita

Paragênese hidrotermal: sericita - clorita calcita - violarita - spionkopita.

\section{CONCLUSÕES}

Os resultados obtidos por este estudo foram inovadores pois não existiam ainda estudos publicados com análises dos sulfetos realizadas pelos métodos de EDS-MEV ou EMPA-WDS no Complexo Gabroico Canindé. Assim, o presente trabalho foi de grande importância na 
compreensão da química mineral.

As principais contribuições deste estudo são:

A identificação de dois minerais ainda não descritos para estas ocorrências, a violarita $\left(\mathrm{FeNi}_{2} \mathrm{~S}_{4}\right)$ e a spionkopita $\left.\left(\mathrm{Cu}_{39} \mathrm{~S}_{28}\right)\right)$. Estes minerais acessórios são resultado de alteração e reconcentração por processos hidrotermais supergênicos.

A descrição dos processos físico-químicos de alteração hidrotermal supergênica que por oxidação dos sulfetos principais levaram a uma reconcentração importante em $\mathrm{Cu}$ e Ni. A importância destes processos na reconcentração de metais críticos pode ser notável se ocorrer a grande escala, como acreditamos ser possível no CGC.

A definição de sequência de cristalização e paragêneses minerais para o CGC.

A conscientização de que o Complexo Gabroico Canindé apresenta uma profusão de ocorrências minerais de considerável potencial econômico, destacando-se aqui o Cu-Ni.

\section{AGRADECIMENTOS}

O presente trabalho foi realizado com apoio da Coordenação de Aperfeiçoamento de Pessoal de Nível Superior - Brasil (CAPES) - Código de Financiamento 001; Fábio Damasceno agradece à CAPES por sua bolsa de mestrado - Código 1764014; ao PGAB e ao CLGeo/UFS pela disponibilidade de sua estrutura e laboratórios; ao Laboratório Nacional de Energia e Geologia (Porto), pelas análises em microssonda eletrônica e ao professor Herbet Conceição, pelo tempo disponibilizado para execução das análises por EDS e aos colegas do RECMIN que de alguma forma ajudaram nesta pesquisa.

\section{REFERÊNCIAS}

BARNES, S.J. \& RIPLEY, E.M. Highly siderophile and strongly chalcophile elements in magmatic ore deposits. Mineral Geochemestry, v. 81, p. 725-774, 2016.

CABRI, L.J. \& LAFLAMME, J.H.G. The mineralogy of the platinum-group elements from some copper-nickel deposits of the Sudbury area, Ontario. Economic Geology, v. 71, p. 11591195,1976

CONCEIÇÃO, J.A.; ROSA, M.L.S.; CONCEIÇÃO, H.; SILVA, C.C., LISBOA, V.A.C. The Glória Sul Stock: An example of a leucogranite in the Macururé Domain, Sergipano Orogenic System, Northeastern Brazil. In: $8^{\mathrm{TH}}$ HUTTON SYMPOSIUM ON GRANITES AND RELATED ROCKS. Resumos Expandidos...Florianopolis, 2015, p. 166.

D'EL REY SILVA, L.J.H. Tectonic Evolution of the Sergipano Belt, NE Brazil. Revista Brasileira de Geociências, v. 25, n. 4, p. 315-332, 1995.

DARE, S.A.S.; BARNES, S.J.; PICHARD, H.P.; FISHER, P.C. Chalcophile and platinum-group element (PGE) concentrations in the sulfide minerals from the McCreedy East deposit, Sudbury, Canada, and the origin of PGE in pyrite. Mineral Deposita, v. 46, p 381-407, 2010.

GOBLE, R.J. The Relationship Between Crystal Structure, Bondingand Cell Dimensions in the Copper Sulfides. Canadian Mineralogist, v. 23, p. 61-76, 1985.

HAWLEY, J.E. \& NICHOL, I. Trace Elements in Pyrite, Pyrrotite and Chalcopyrite of Different Ores. Economic Geology, v. 56, p. 467-487, 1961.

MARQUES DE SÁ, C.D.; MARTINS, B.L.L; BARRETO, D.S.; PAIM, M.; CONCEIÇÃO, H. First finding of native Nickel in cumulates of the Canindé Domain, Brazil. Revista Pincipia, v. 43, p. 166-174, 2018.

MARTINS, B.L.L. Petrografia, geoquímica e mapeamento dos cumulatos de Fe-Ti do Domínio Canindé. São Cristovão, 74 p., 2017. Monografia (Graduação em Geologia) Departamento de Geologia, Universidade Federal de Sergipe.

OLIVEIRA, E.P.; WINDLEY, B.F.; ARAÚJO, M.N.C. The Neoproterozoic Sergipano orogenic belt, NE Brazil: A complete plate tectonic cycle in western Gondwana. Precambrian Research, v. 181, p. 64-84, 2010
SANTOS, R.A. \& SOUZA, J.D. (Coordenadores). Programa Levantamentos Geológicos Básicos do Brasil. Carta geológica, Carta metalogenética/previsional - Escala 1:100.000, Folha SC.24-X-C-VI Piranhas. Convênio Departamento Nacional de Mineração (DNPM)/Serviço Geológico do Brasil (CPRM), Brasília, 154 p., 1988.

SANTOS, R.A. (Coord). Programa Levantamentos Geológicos Básicos do Brasil - PLGB. Geologia e recursos minerais do Estado de Sergipe. Texto explicativo do Mapa geológico do Estado de Sergipe. Escala 1:250.000. Brasília: Convenio CPRM/DIEDIG/DEPAT; CODISE, 156 p., 2001.

SEIXAS, S.R.M. \& DEMORAES, L.C. Projeto Canindé. Exame Atualizado de Projeto-EXAP. Convênio Serviço Geológico do Brasil (CPRM)/DRM/DEREM, Salvador, 21 p., 1996.

SILVA FILHO, M.A. (Coord.). Projeto Baixo São Francisco/Vaza-Barris. Relatório final. Salvador: Serviço Geológico do Brasil (CPRM), v. 19, 1977.

SILVA FILHO, M.A. (Coordenador.). Projeto Complexo Canindé do S. Francisco. Relatório Final de Geologia. Salvador: Serviço Geológico do Brasil (CPRM), v.1, 189 p., 1979.

TEIXEIRA, L. Mapa geológico e de recursos minerais do Estado de Sergipe. Escala 1:250.000, 2014.

TENAILLEAU, C.; PRING, A.; ETSCHMANN, B.; BRUGGER, J.; GRGURIC, B.; PUTNIS, A. The transformation of pentlandite to violarite under mild hydrothermal conditions. American Mineralogist, v 91, p. 706-709, 2006.

TESCH, N.A. (Coord.). Projeto Canindé. Relatório de pesquisa. Salvador: Serviço Geológico do Brasil (CPRM), v. 4, 200 p., 1980. 